\title{
CORRELACIÓN ANATOMOPATOLÓGICA DE LAS HIPERPLASIAS ENDOMETRIALES ANTES Y DESPUÉS DE LA HISTERECTOMÍA
}

\author{
Raúl Barrero P. ${ }^{1}$, David Barrero V. a, Catalina Alonso M. 1, Gladys García M. 2, \\ Victoria Vargas V. ${ }^{a}$, René Rivera Z. ${ }^{1}$, Angélica Larraín $H .{ }^{b}$ \\ ${ }^{1}$ Servicio de Ginecología y Obstetricia, ${ }^{2}$ Servicio de Anatomía Patológica, Hospital Clínico Félix Bulnes.
}

${ }^{a}$ Interno de Medicina, Universidad de Chile. ${ }^{b}$ Matrona, Escuela de Obstetricia, Universidad de Santiago de Chile

\begin{abstract}
RESUMEN
Objetivo: Correlacionar histológicamente las biopsias del legrado uterino con las biopsias de las piezas de histerectomía en pacientes con hiperplasia endometrial (HE). Método: Se diagnosticaron con biopsia de legrado uterino 90 pacientes con HE entre enero de 2001 y diciembre de 2005. De estas pacientes, 46 correspondieron a HE con atipia (grupo 1) y 44 a HE sin atipia (grupo 2). Todas las pacientes del grupo 1 se sometieron a histerectomía total más salpingooforectomía bilateral. A 28 pacientes del grupo 2 se les realizó la misma cirugía por patologías ginecológicas asociadas. Se compararon los resultados de las biopsias pre y postoperatorias de las 74 pacientes operadas, evaluándose la concordancia entre ellas. Resultados: En la biopsia de la pieza de histerectomía del grupo 1 se observan 31 casos con HE con atipia $(67,4 \%), 13$ casos $(28,3 \%)$ sin atipias y 2 casos $(4,3 \%)$ de cáncer endometrial. En el grupo 2 hubo 16 casos $(57,1 \%)$ con $\mathrm{HE}$ sin atipia, 10 casos $(35,7 \%)$ con endometrio normal y $2(7,1 \%)$ casos de HE con atipia. La concordancia fue de un $63 \%(p=0,000)$ entre ambas biopsias y resultó significativamente más baja en el subgrupo de pacientes que presentaban atipias en la biopsia preoperatorio, respecto a las pacientes $\sin$ atipias $(p=0,028)$. El likehood ratio de la biopsia preoperatorio de pacientes con HE con atipias fue de 33,2. Conclusión: El diagnóstico con biopsia preoperatoria por legrado, de las pacientes con HE, tuvo una precisión aceptable en comparación a la biopsia de la pieza operatoria, apoyando su utilidad en el manejo de estas pacientes.
\end{abstract}

\section{PALABRAS CLAVE: Hiperplasia endometrial, histerectomía, biopsia}

\section{SUMMARY}

Objective: To evaluate the hystopathologic correlation between curettage and hysterectomy specimens in patients with endometrial hyperplasia. Methods: 90 patients were diagnosed with endometrial hyperplasia in curettage specimens between January 2001 and December 2005. Of these patients 46 were found to have atypical hyperplasia (group 1) and 44 hyperplasia without atypias (group 2). All the patients in group 1 had a total hysterectomy plus bilateral anexectomy; 28 patients of group 2 had the same surgery because of associate gynecological pathology. Curettage and hysterectomy specimens of 74 patients were compared and evaluated the concordance between them. Results: Of hysterectomy specimens in group 1, 31 cases had atypical hyperplasia (67.4\%), 13 cases $(28.3 \%)$ hyperplasia without atypias and 2 cases $(4.3 \%)$ with endometrial carcinoma. In group 2, 16 cases $(57.1 \%)$ of hyperplasia without atypias, 10 cases $(35.7 \%)$ with normal endometrium and $2(7.1 \%)$ cases of atypical hyperplasia were found. The agreement of the hystopathological diagnosis of endometrial hyperplasia between both biopsies was $63 \%(p=0.000)$ and it was 
significantly lower in the subgroup of patients that had atypias on the curettage biopsy with respect to the patients with hyperplasia with no atypias $(\mathrm{p}=0.028)$. The likehood ratio of the biopsy by curettage of patients with atypias was of 33.2. Conclusion: The accuracy of the curettage biopsies as compared with hysterectomy specimens in patients with endometrial hyperplasia was acceptable, supporting its usefulness in the management of these patients.

\section{KEY WORDS: Endometrial hyperplasia, hysterectomy, biopsy}

\section{INTRODUCCIÓN}

La hiperplasia endometrial (HE) es un diagnóstico frecuente dentro de la ginecología general (1). Se caracteriza por la proliferación de las glándulas y del estroma endometrial secundario al estímulo estrogénico permanente (2). Se ha visto que el endometrio hiperplásico puede regresar a la normalidad en forma espontánea o con tratamiento, persistir como tal o evolucionar a carcinoma (3).

La HE se clasifica en: a. simple y compleja sin atipia y b. simple y compleja con atipia (4). Las atipias celulares pueden ser leves, moderadas y severas, siendo estas últimas las que más preocupan por su relación con el cáncer endometrial (CE) tipo endometrioide (5).

El engrosamiento endometrial, el sangrado uterino anormal y los factores de riesgo para CE obligan a realizar biopsia de endometrio (6-10).

Uno de los problemas descritos en la literatura relacionados con la hiperplasia endometrial es la dificultad que existe para diagnosticar y distinguir entre los distintos subgrupos que la componen, principalmente entre la hiperplasia compleja atípica y el carcinoma endometrial $(11,12)$.

El objetivo de este estudio es correlacionar histológicamente las biopsias del legrado uterino con las biopsias de las piezas de histerectomía en pacientes con HE.

\section{PACIENTES Y MÉTODO}

En el Servicio de Ginecología y Obstetricia del Hospital Félix Bulnes, entre el 1 de enero de 2001 y el 31 de diciembre de 2005, se diagnosticó hiperplasia endometrial en 90 pacientes mediante muestras obtenidas con legrado uterino. De estas, 46 correspondieron a hiperplasias con atipia (grupo 1) y 44 a hiperplasias sin atipias (grupo 2). Todas las pacientes del grupo 1 se sometieron a histerectomía total (HTT) más salpingectomía y ooforectomía bilateral (SOB). De las pacientes del grupo 2 sólo en 28 se realizó HTT más SOB, y en los 16 casos restantes se realizó exclusivamente tratamiento médico con progestinas. De las 74 pacien- tes operadas, se revisaron retrospectivamente las fichas clínicas y se registraron los siguientes datos: edad, paridad, presencia de diabetes mellitus, presencia de hipertensión arterial, grosor endometrial en la ecografía transvaginal, patologías ginecológicas asociadas y los resultados de las biopsias del legrado uterino y de la pieza de histerectomía. Se compararon los resultados de ambas biopsias y se evaluó la concordancia que hubo entre ellas. Se utilizó la clasificación de la Sociedad Internacional de Patólogos Ginecológicos según los criterios de la Organización Mundial de la Salud.

Los análisis estadísticos se efectuaron utilizando el software STATA 7.0 utilizando la prueba de Chi cuadrado para comparar las proporciones de variables nominales entre los dos grupos. La concordancia entre los resultados de la biopsia por legrado uterino y la biopsia operatoria se realizó mediante la prueba $V$ de Cramer. Se estableció como nivel de significación estadística un $\square=0,05$.

\section{RESULTADO}

De las 74 pacientes con HE estudiadas, la media de edad fue 47,9 años ( $D E \pm 6,23$ años). En relación a la paridad un $97,3 \%$ de las pacientes era multípara. La media del peso fue de $74,1 \mathrm{~kg}$ (DE $\pm 13,4 \mathrm{~kg}$ ). El grosor endometrial en la ecografía transvaginal tuvo un promedio de $13,1 \mathrm{~mm}$ (DE \pm $6,1 \mathrm{~mm}$ ). El $13,5 \%$ de las pacientes tenía el diagnóstico de diabetes mellitus y el $45,9 \%$ de hipertensión arterial crónica, siendo el $6,8 \%$ portadoras de ambas patologías. De las patologías ginecológicas asociadas más frecuentes la miomatosis estuvo presente en $32,4 \%$ de los casos y la adenomiosis en $25,7 \%$. Hubo un $27 \%$ de pacientes que no tuvieron patologías ginecológicas asociadas, y que se operaron solo por la presencia de la hiperplasia (Tabla I).

En la biopsia preoperatoria de las pacientes incluidas en el estudio hubo 46 casos con HE con atipia (16 simples y 30 complejas) y 28 casos con HE sin atipias (15 simples y 13 complejas). Los resultados de la biopsia de la pieza de histerectomía informó 22 muestras con HE sin atipias (16 simples 
Tabla I

CARACTERÍSTICAS CLÍNICAS DEL GRUPO DE PACIENTES CON HIPERPLASIA ENDOMETRIAL

\begin{tabular}{|c|c|c|}
\hline Variable & & \\
\hline Edad (años) & $47,9 \pm 6,23$ & \\
\hline Peso promedio $(\mathrm{kg})$ & $74,1 \pm 13,4$ & \\
\hline \multirow[t]{6}{*}{ Paridad } & Nuligesta (\%): & 2,7 \\
\hline & Multípara (\%): & \\
\hline & $1-2$ & 35,1 \\
\hline & $3-4$ & 48,6 \\
\hline & $5-6$ & 10,8 \\
\hline & 7 & 2,7 \\
\hline Hipertensión arterial (\%) & 45,9 & \\
\hline Diabetes mellitus (\%) & 13,2 & \\
\hline Grosor endometrial (mm) & $13,1 \pm 6,1$ & \\
\hline \multirow[t]{6}{*}{ Patologías ginecológicas asociadas (\%) } & Sin patologías & 27,0 \\
\hline & Miomatosis & 32,4 \\
\hline & Adenomiosis & 25,7 \\
\hline & Pólipo endometrial & 9,5 \\
\hline & Quiste ovárico & 4,1 \\
\hline & Ovario poliquístico & 1,4 \\
\hline
\end{tabular}

y 6 complejas), 33 muestras con HE con atipia (16 simples y 17 complejas), 2 cánceres de endometrio y 17 casos con endometrio informado como normal (Tabla II).

Del grupo de pacientes con HE con atipia en la biopsia preoperatoria, $31(67,4 \%)$ persistieron con el mismo diagnóstico en la pieza operatoria; en 13 pacientes $(28,3 \%)$ se observó ausencia de atipias en la pieza de histerectomía y en $2(4,3 \%)$ casos se hizo el diagnóstico de cáncer endometrial (Tabla III). De las pacientes con HE con atipia en la biopsia preoperatoria se observa que el $86,9 \%$ corresponden a atipias leves, el 6,5\% a atipias moderadas y sólo el 4,4\% a severas. En los resultados del grado de atipia en las biopsias postoperatorias destaca una disminución del porcentaje de atipias leves en un $19,5 \%$ y la ausencia de atipias moderadas, la aparición de $2(4,4 \%)$ cánceres de endometrio, correspondientes a los casos de atipias severas (1 HE simple y $1 \mathrm{HE}$ compleja), y la ausencia de atipias en 13 casos $(28,3 \%)$ ( $7 \mathrm{HE}$ grado leve y $6 \mathrm{HE}$ grado moderado) (Tabla IV).

Al analizar lo que ocurrió en el grupo de 28 pacientes con HE sin atipias, observamos que en
26 casos se mantuvo la ausencia de atipia; de las cuales $10(35,7 \%)$ son endometrios normales y 16 $(57,15 \%) \mathrm{HE}$ y en 2 pacientes $(7,1 \%)$ se diagnosticó la presencia de atipias (Tabla III). La Tabla V muestra esta distribución según la HE fuesen simples o complejas.

Agrupando la presencia de HE con atipias o cáncer como biopsias "patológicas" y las biopsias informadas como con HE sin atipias o normales como "no patológicas", la concordancia entre las biopsias preoperatorias y las de la pieza operatoria fue de $63 \%$ según la prueba $V$ de Cramer $(p=0,000)$ (Tabla VI). Esta concordancia fue significativamente menor en el subgrupo de pacientes que presentaban atipias en la biopsia preoperatorio comparado con las que no presentaban atipias la prueba de Chi cuadrado $(p=0,028)$ (Tabla VII). Si se considera la biopsia de la pieza operatoria como el diagnóstico definitivo de la presencia o ausencia de la enfermedad, los valores de sensibilidad y especificidad para biopsia preoperatoria resultaron ser de $94 \%$ y $67 \%$ respectivamente. El likelihood ratio de la biopsia preoperatoria con atipias fue de 33,2. 
Tabla II

TIPOS HISTOPATOLÓGICOS DE HIPERPLASIA ENDOMETRIAL EN LAS BIOPSIAS POR LEGRADO UTERINO SEGMENTARIO Y DE LA PIEZA DE HISTERECTOMÍA

\begin{tabular}{|c|c|c|c|c|c|c|c|}
\hline & $\begin{array}{l}\text { HES } \\
\sin A\end{array}$ & $\begin{array}{l}\mathrm{HEC} \\
\sin A\end{array}$ & $\begin{array}{l}\text { HES } \\
\text { con A }\end{array}$ & $\begin{array}{r}\text { HEC } \\
\operatorname{con} A\end{array}$ & Cáncer & $\begin{array}{c}\text { Endometrio } \\
\text { normal }\end{array}$ & Total \\
\hline $\begin{array}{l}\text { Biopsia por } \\
\text { legrado } \\
\text { uterino }\end{array}$ & $\begin{array}{c}15 \\
(20,2 \%)\end{array}$ & $\begin{array}{c}13 \\
(17,6 \%)\end{array}$ & $\begin{array}{c}16 \\
(23,0 \%)\end{array}$ & $\begin{array}{c}30 \\
(39,2 \%)\end{array}$ & - & - & 74 \\
\hline $\begin{array}{l}\text { Biopsia pieza } \\
\text { histerectomía }\end{array}$ & $\begin{array}{c}16 \\
(21,6 \%)\end{array}$ & $\begin{array}{c}6 \\
(8,1 \%)\end{array}$ & $\begin{array}{c}16 \\
(21,6 \%)\end{array}$ & $\begin{array}{c}17 \\
(23,0 \%)\end{array}$ & $\begin{array}{c}2 \\
(2,7 \%)\end{array}$ & $\begin{array}{c}17 \\
(23,0 \%)\end{array}$ & 74 \\
\hline Total & 31 & 19 & 33 & 46 & 2 & 17 & \\
\hline
\end{tabular}

HE: hiperplasia endometrial. A: atipias. S: simple. C: compleja.

Tabla III

EVALUACIÓN DE LA BIOPSIA PRE Y POSTOPERATORIA EN PACIENTES CON HIPERPLASIA ENDOMETRIAL

\begin{tabular}{|c|c|c|c|c|}
\hline \multirow{4}{*}{$\begin{array}{l}\text { Biopsia por } \\
\text { legrado uterino }\end{array}$} & \multicolumn{4}{|c|}{ Biopsia pieza histerectomía } \\
\hline & HE sin atipia y & HE con atipia & Cáncer & Total \\
\hline & endometrio sano & & & \\
\hline & n (\%) & $\mathrm{n}(\%)$ & $\mathrm{n}(\%)$ & \\
\hline HE sin atipia & $26(92,9 \%)$ & $2(7,1 \%)$ & - & 28 \\
\hline HE con atipia & $13(28,3 \%)$ & $31(67,4 \%)$ & $2(4,3 \%)$ & 46 \\
\hline Total & 39 & 33 & 2 & 74 \\
\hline
\end{tabular}

HE: hiperplasia endometrial

\section{DISCUSIÓN}

La concordancia global encontrada en este trabajo entre la biopsia pre y postoperatoria fue de $63 \%$, lo que implica una discordancia o error de $37 \%$. Sin embargo, cuando las atipias estuvieron presentes se obtuvo un likelihood ratio de 33,2 para las biopsias por legrado uterino, lo que quiere decir que la probabilidad de que las atipias efectivamente estén presentes es significativa.

Todos los otros trabajos publicados que correlacionan ambas biopsias son series de casos que comparan los resultados de las biopsias preoperatorios con la biopsia de la pieza operatoria en pacientes con HE $(13,14,15)$. Estos estudios informan diversos resultados en la concordancia entre ambas biopsias con variaciones entre el 30 a $89 \%$ depen- diendo el tipo de hiperplasia, siendo las sin atipias las tendientes a ser más altas, debido a una alta presencia de cáncer en las piezas operatorias de legrados informados como con atipias solamente.

Las diferencias entre los distintos estudios, incluyendo el actual, podrían deberse a la forma de obtener la muestra endometrial y a las discrepancias en la evaluación anatomopatológica de los distintos centros. En los casos donde hubo ausencia de HE o donde las hiperplasias con atipias no presentaron atipias en la muestra de la histerectomía, la explicación podría ser la total remoción del tejido comprometido en el curetaje o bien un sobrediagnóstico en las biopsias preoperatorias.

Una de las diferencias más importantes al comparar las biopsias pre y postoperatorias entre los distintos trabajos está en el porcentaje de casos con 
Tabla IV

GRADOS DE ATIPÍA DE LA HIPERPLASIA ENDOMETRIAL PRE Y POSTQUIRÚRGICA

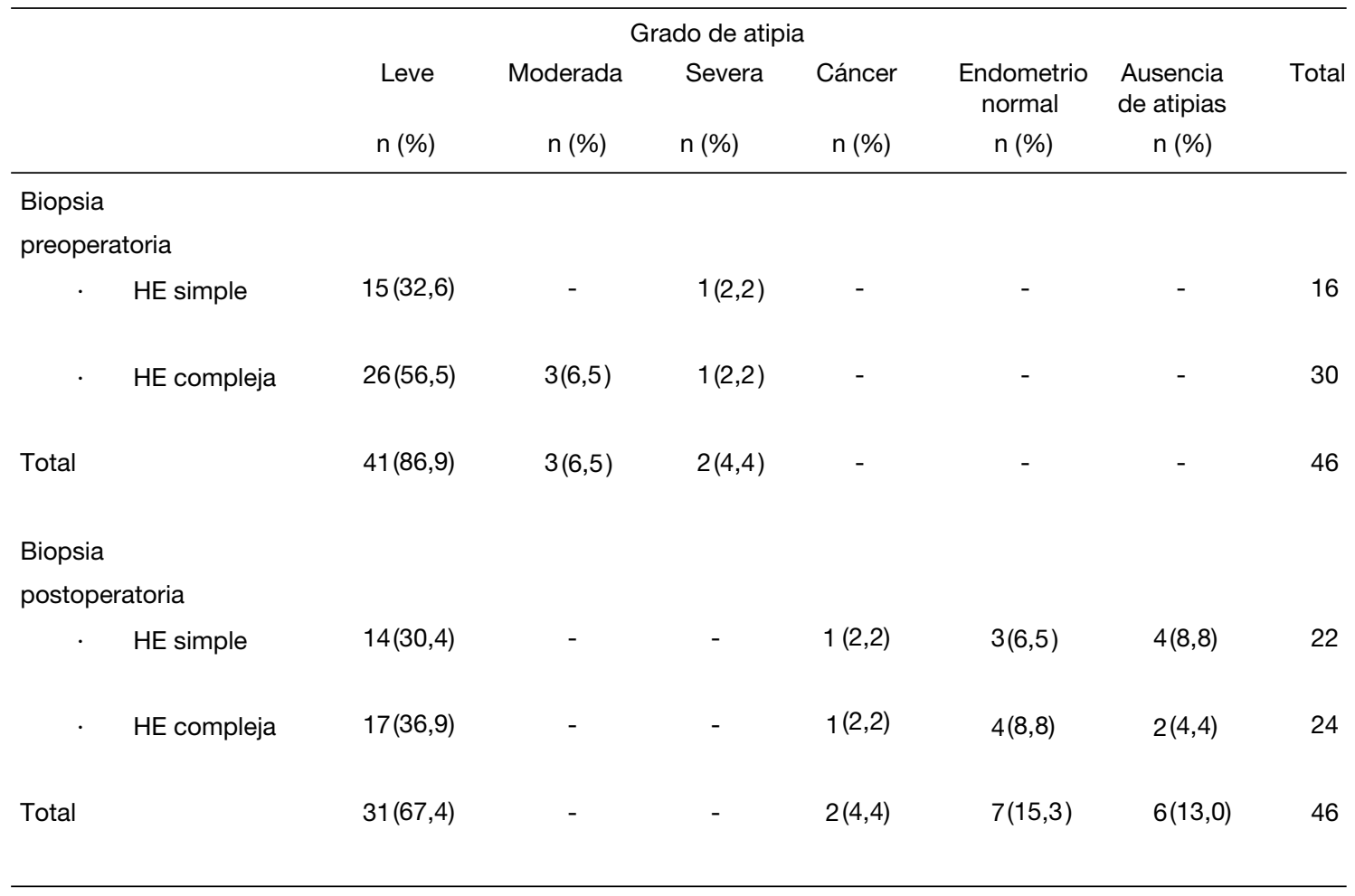

HE: hiperplasia endometrial.

Tabla V

EVALUACIÓN DE LA HIPERPLASIA ENDOMETRIAL SIN ATIPIAS DESPUÉS DE LA HISTERECTOMÍA

\begin{tabular}{|c|c|c|c|c|}
\hline & $\begin{array}{c}\text { Endometrio } \\
\text { normal } \\
\text { n (\%) }\end{array}$ & $\begin{array}{c}\mathrm{HE} \\
\text { sin atipia } \\
\mathrm{n}(\%)\end{array}$ & $\begin{array}{c}\text { Endometrio } \\
\text { con atipia } \\
\text { n (\%) }\end{array}$ & Total \\
\hline HE simple & $3(10,7)$ & $10(35,7)$ & $2(7,1)$ & 15 \\
\hline HE compleja & $7(25,0)$ & $6(21,4)$ & - & 13 \\
\hline Total & $10(35,7)$ & $16(57,1)$ & $2(7,1)$ & 28 \\
\hline
\end{tabular}

HE: hiperplasia endometrial.

cáncer endometrial subdiagnosticado en la biopsia preoperatoria (13-16). Existe un alto porcentaje de cáncer concomitante en pacientes con $\mathrm{HE}$ con y sin atipia, aunque la prevalencia en $\mathrm{HE}$ con atipia es mayor, variando entre $17 \%$ a $52 \%$. De los trabajos reportados que comparan los resultados de las biopsias pre y postoperatorias con el objetivo de evaluar la presencia de carcinoma endometrial concomitante existe sólo un estudio de cohorte prospectivo publicado el año 2006 por el Grupo Oncológico Ginecológico (GOG). En este trabajo se evaluó la prevalencia de carcinoma endometrial concomitante en pacientes con HE con y sin atipia, encontrándose un $39,1 \%$ y $18,9 \%$, respectivamente 
Tabla VI

CONCORDANCIA ENTRE LAS BIOPSIAS PREOPERATORIAS Y LAS DE LA PIEZA DE HISTERECTOMÍA EN PACIENTES CON HE

\begin{tabular}{lccc}
\hline Biopsia por legrado uterino & \multicolumn{2}{c}{ Pieza histerectomía } & Total \\
& Con patologías & Sin patologías & 46 \\
Con patologías & 33 & 13 & 28 \\
Sin patologías & 22 & 6 & 74 \\
\hline Total & 35 & 39 & 28 \\
\hline
\end{tabular}

Concordancia 63\%. Sensibilidad: 94\%. Especificidad: 67\%. Likelihood ratio: 33,2.

Tabla VII

DIFERENCIA DE LAS CONCORDANCIA ENTRE LA BIOPSIA PRE Y POSTOPERATORIA SEGÚN LA PRESENCIA O NO DE ATIPIA

\begin{tabular}{lccc}
\hline & Con patología & Sin patología & Total \\
\hline $\begin{array}{l}\text { Discordancia entre la biopsia } \\
\text { pre y postoperatoria }\end{array}$ & 13 & 2 & 15 \\
\end{tabular}

Concordancia entre la biopsia

33

26

59

pre y postoperatoria

\begin{tabular}{llll}
\hline Total & 46 & 28 & 74 \\
\hline$p=0,028$. & & &
\end{tabular}

(16). Es destacable que el diagnóstico preoperatorio se realizó mediante distintos métodos diagnósticos, tales como legrado uterino, cánula Novak, Vabra y Pipelle, a diferencia del trabajo actual donde el diagnóstico fue realizado siempre con legrado uterino segmentario.

En este estudio la concomitancia de carcinoma endometrial fue de un $4,3 \%$ en el grupo de $\mathrm{HE}$ con atipia y no se encontraron casos en el grupo de HE sin atipia. Esta importante diferencia con los otros estudios podría explicarse por las razones expuestas anteriormente, pero también en parte por las diferencias propias de las distintas poblaciones, como por ejemplo las diferencias étnicas y el hecho de que el cáncer de endometrio sea una patología más frecuente en países desarrollados donde existe mayor prevalencia de factores de riesgo como son la obesidad, la diabetes mellitus, etc.

Los dos casos de HE con atipia donde se diagnosticó un carcinoma endometrioide concomitante en la biopsia postoperatoria, correspondieron a atipias severas y a carcinomas endometrioides bien diferenciados, etapa Ib y la. En ambos casos des- taca la demora en la realización de la cirugía. En el primer caso este retraso fue de 20 meses debido a una cardiopatía descompensada y bronquitis obstructiva crónica, y en la segunda paciente se realizó inicialmente una polipectomía con atipia severa en otro centro, decidiéndose en el comité de ginecología oncológica repetir la biopsia. Esta última es informada como HE sin atipias y se realiza manejo médico que fracasa. La paciente es operada 33 meses después con el hallazgo histológico ya descrito.

En el grupo de pacientes con $\mathrm{HE}$ sin atipia hubo 2 casos donde se encontraron atipias, lo que podría deberse al curetaje incompleto de la cavidad endometrial, al muestreo insuficiente de la biopsia preoperatorio o al desarrollo de atipias entre el legrado y la histerectomía.

Existe consenso en el tratamiento quirúrgico de la $\mathrm{HE}$ con atipia, pero debido a la discordancia reportada entre la biopsia diferida preoperatoria y la pieza operatoria, que también podría existir entre una biopsia rápida y diferida postoperatoria, es que la biopsia rápida no se recomienda para la toma de 
decisiones en el manejo de estas pacientes (17). Este razonamiento podría hacer cuestionar la conducta de incluir una biopsia rápida de la pieza de histerectomía para descartar la presencia de un carcinoma endometrioide concomitante cuando se operan pacientes con HE con atipias (principalmente severas), con el objeto de realizar la cirugía en un tiempo. Sería importante entonces enfatizar en el diagnóstico preoperatorio lo más certero posible, apoyándose en otros exámenes diagnósticos como por ejemplo la histeroscopia.

Este trabajo también apoya el tratamiento médico establecido para las HE sin atipia, por su alta concordancia con la biopsia postoperatoria y la ausencia de cáncer en estas pacientes.

\section{CONCLUSIÓN}

En este estudio la biopsia pre y postoperatoria de las pacientes con HE presenta un importante porcentaje de concordancia, con una altísima probabilidad post-test para biopsias preoperatorias patológicas, apoyando su utilidad en la toma de decisiones del tratamiento más adecuado y oportuno de las pacientes.

\section{BIBLIOGRAFÍA}

1. Jiménez J, Hiriart J, Coda S, Corvalán J, Olivares M, Zamora E, et al. Experiencia clínica en histeroscopía diagnostica ambulatoria: Análisis 1.312 casos. Rev Obstet Ginecol Hospital Santiago Oriente Dr. Luis Tisné Brousse 2006;1(3):171-3.

2. Philip J, Disaia, William T, Creasman. Hiperplasia endometrial, tratamiento estrogénico. Oncología ginecológica clínica. 4⿳亠丷厂 edición. España: Editorial Mosby / Doyma libros, 1994;126-55.

3. Terakawa N, Kigawa J, Taketani Y, et al. The behavior of endometrial Hyperplasia: a prospective study. Endometrial Hyperplasia Study group. J Obstet Gyanecol 1997;23:223-30.

4. Ronnet BM, Kurman RJ. Precursor lesions of endometrial carcinoma. Kurman RJ (ed). Blausteins Pathology of the female genital tract. $5^{\mathrm{a}}$ ed. New York: Springer. Veriag, 2002;467-500.
5. Montgomery B, Daum G, Dunton. Endometrial Hyperplasia: A review. Obstet Ginaecol Surv 2004;59(5):36878.

6. Dominguez C, Zamora J, Barrera S, Tacla X. Biopsia endometrial ambulatoria: experiencia preliminar. Rev Chil Obstet Ginecol 2006;71(3):161-4.

7. Bianchi M, Berrios C, Villaseca P, Arteaga E. Patología endometrial en mujeres con sangrado anormal durante terapia de reemplazo hormonal. Rev Chil Obstet Ginecol 2003;68(6)487-90.

8. Kurman RJ, Kaminski PF, Moris HJ. The behavior of endometrial hyperplasia. A long term study of untreated hyperplasia in 170 patients. Cancer (Phil) 1985;56:40312.

9. Sivridis E, Giatromanolaki A. Prognostic aspects on endometrial hyperplasia and neoplasia. Virchows Arch 2001;439:118-26.

10. Nazzal O, Suárez E, San Martín R, Celis M. Cáncer de endometrio En: Eghon Guzmán. Selección de temas en Gineco-obstetricia. $1^{\circ}$ ed Santiago: Editorial Publimpact 2005;555-69.

11. Zaino R, Kauderer PF, Trimble CL, et al. Reproducibility of the diagnosis of atypical endometrial hyperplasia: a gynaecologic oncology group study. Cancer 2006;106(4):804-11.

12. Kendall BS, Ronnett BM, Isacson C, et al. Reproducibility of the diagnosis of endometrial hyperplasia, atypical hyperplasia and well differentiated carcinoma. Am J Surg Pathol 1998;22:1012-9.

13. Gudem G, Sendag F, Kazandi M, et al. Preoperative and postoperative correlation of histopathological findings in cases of endometrial hyperplasia. Eur J Gynaecol Oncol 2003;24(3-4):330-3.

14. Jesadapatrakul S, Tangjitgamol S, Manusirivitaya S. Histopathologic consistency between endometrial hyperplasia diagnosis from endometrial curettage and pathologic diagnoses from hysterectomy specimens. J Med Assoc Thai 2005;88 Suppl 2:S16-21.

15. Dordević B, Stanojević Z, Zivković V, Lalosević D, Gligorijević J, Krstić M. Preoperative and postoperative histopathological findings in patients with endometrial hyperplasia. Med Pregl 2007;60(7-8):372-6.

16. Trimble C, Kauderer J, Zaino R, et al. Concurrent endometrial carcinoma in women with a biopsy diagnosis of atypical endometrial hyperplasia. Cancer 2006;106(4):812-9.

17. Sorosky J. Endometrial Cancer. Obstet Gynecol 2008;111:436-47. 